\title{
PERITÔNIO BOVINO CONSERVADO NA CORREÇÃO DE HÉRNIA VENTRAL EM RATOS: UMA ALTERNATIVA PARA TELA CIRÚRGICA BIOLÓGICA
}

\author{
PRESERVED BOVINE PERITONEUM FOR VENTRAL HERNIA REPAIR IN RATS: \\ A BIOLOGICAL SURGICAL MESH ALTERNATIVE
}

\author{
Eduardo Lemos de Souza Bastos ${ }^{1}$; Djalma José FagundesTCBC-SP²; \\ Murched Omar Taha, TCBC-SP'; Neil Ferreira Novo ${ }^{4}$; Rubens Augusto Brazil Silvado ${ }^{5}$
}

\begin{abstract}
RESUMO: Objetivo: Verificar a possibilidade de implantação e a capacidade de resistência tênsil do peritônio parietal bovino como tela cirúrgica na correção de hérnia ventral em um modelo animal de experimentação. Método: Utilizando 57 ratos machos Wistar, comparou-se o implante do peritônio bovino com a tela de polipropileno na correção de um defeito provocado na parede abdominal do animal. Após sete (sub-grupo A) e 28 (sub-grupo B) dias de observação, as peças foram retiradas e procedeu-se a avaliação da resistência à tração em Máquina Universal de Ensaios. Um grupo sem implante de material protético foi utilizado como controle nos testes de força tênsil. Os testes de Mann-Whitney e de Kruskal-Wallis foram utilizados e estabeleceu-se em 0,05 o nível para rejeição da hipótese de nulidade. Resultados: Os testes de resistência à tração, com valores expressos em Newton, não mostraram diferenças estatísticas entre os grupos estudados, tanto no $7^{\circ}$ quanto no $28^{\circ}$ dia de pós-operatório, e ambos foram menos resistentes que a parede abdominal normal $(\mathrm{p}=0,003)$. Conclusão: O peritônio parietal bovino apresentou resistência tênsil semelhante a da tela de polipropileno em um modelo de correção de hérnia ventral em ratos (Rev. Col. Bras. Cir. 2005; 32(5): 256-260).
\end{abstract}

Descritores: Hérnia ventral; Telas cirúrgicas; Peritônio; Modelos animais.

\section{INTRODUÇÃO}

Pioneiramente relatada por Usher et $_{\text {al. }}{ }^{1}$, a utilização de telas cirúrgicas na correção de hérnias abdominais recebeu um grande impulso a partir do estabelecimento do conceito "tension-free" 2 e da associação com um menor índice de recidivas ${ }^{3,4}$. As telas absorvíveis, sintéticas ou biológicas, apresentam vantagens inerentes à sua característica de absorção pelo organismo. Entre as próteses absorvíveis biológicas mais estudadas e utilizadas estão: a dura-máter humana ${ }^{5,6}$, a membrana amniótica humana ${ }^{7,8}$, o saco herniário humano ${ }^{9,10}$, a fáscia-lata humana ${ }^{11,12}$, matrizes extracelulares de colágeno suíno ${ }^{13-15}$ e o pericárdio de bovinos ${ }^{16,17}$, eqüinos ${ }^{18}$ ou caprinos ${ }^{19}$.

O pericárdio bovino é uma prótese biológica de uso corrente mas apresenta limitação na extensão do tecido obtido para implante. Baseado na experiência da medicina veterinária surgiu a idéia do uso do peritônio parietal bovino ${ }^{20,21}$. Esta membrana, que pode ser captada e submetida ao mesmo preparo consagrado para o pericárdio bovino, fornece uma área maior de tecido aproveitável e adaptável às diversas situações da prática cirúrgica.

Os objetivos do presente estudo foram os de verificar a possibilidade de implantação e a capacidade de resis- tência tênsil do peritônio parietal bovino como tela cirúrgica na correção de hérnia ventral em um modelo animal de experimentação.

\section{MÉTODO}

O experimento foi aprovado pela Comissão de Ética em Pesquisa da Universidade Federal de São Paulo (UNIFESP-EPM) sob o protocolo $n^{\circ}$ 280/01. A amostra foi constituída por 57 ratos machos, adultos, Wistar, provenientes do Biotério da Faculdade de Medicina de Marilia (FAMEMA), alojados individualmente em gaiolas, mantidos sem restrição alimentar, sob iluminação controlada (ciclo claro/escuro de 12 horas), temperatura ambiente e em condições adequadas de higiene, com um período pré-operatório de adaptação de sete dias.

Os animais do experimento foram distribuídos em três grupos, conforme o material utilizado para correção da hérnia ventral:

Grupo 1 (20 animais): correção da hérnia ventral com peritônio bovino

$$
\text { Subgrupo A }(\mathrm{n}=10) \text { : } \text { morte no } 7^{\circ} \mathrm{PO}
$$

Subgrupo $\mathrm{B}(\mathrm{n}=10)$ : morte no $28^{\circ} \mathrm{PO}$

\footnotetext{
1. Docente da Disciplina de Cirurgia do Sistema Digestório da Faculdade de Medicina de Marília (FAMEMA); Pós-Graduando (nível Doutorado) do Programa de Cirurgia e Experimentação da Universidade Federal de São Paulo - Escola Paulista de Medicina (UNIFESP-EPM).

2. Professor Adjunto da Disciplina de Técnica Operatória e Cirurgia Experimental do Departamento de Cirurgia da UNIFESP-EPM; Coordenador do Programa de Pós-Graduação em Cirurgia e Experimentação da UNIFESP-EPM.

3. Professor Afiliado da Disciplina de Técnica Operatória e Cirurgia Experimental do Departamento de Cirurgia da UNIFESP-EPM.

4. Professor Titular do Departamento de Saúde Coletiva da Universidade de Santo Amaro (UNISA).

5. Chefe da Disciplina de Cirurgia do Sistema Digestório da FAMEMA; Diretor de Pós-Graduação da FAMEMA.
}

Recebido em: 23/03/2005

Aceito para publicação em: 09/09/2005

Conflito de interesse: nenhum

Fonte de financiamento: CAPES/PRPG

Trabalho realizado na Universidade Federal de São Paulo. 
Grupo 2 (19 animais): correção da hérnia ventral com tela de polipropileno

Subgrupo A $(n=10)$ : morte no $7^{\circ} \mathrm{PO}$

Subgrupo B $(n=9)$ : morte no $28^{\circ} \mathrm{PO}$

Grupo 3 (17 animais): nenhum material protético.

Subgrupo A ( $\mathrm{n}=9)$ : morte no $7^{\circ} \mathrm{PO}$

Subgrupo B $(n=8)$ : morte no $28^{\circ} \mathrm{PO}$

Preparo do peritônio bovino: após o abate de um animal sadio (com finalidade de descarte e estudo) nas dependências do Hospital Veterinário da Disciplina de Medicina Veterinária e Zootecnia da Universidade de Marília, o peritônio parietal foi retirado através da dissecação com tesoura romba e pinça dente-de-rato e imediatamente imerso em um recipiente contendo solução salina de cloreto de sódio a $0,9 \%$ (soro fisiológico) por um período de 10 minutos. Na seqüência, foi lavado em água corrente e retirados os excessos de gorduras e fáscias adjacentes, produzindo uma membrana macia e de cor amarelada, que foi então recortada em porções retangulares de aproximadamente $10 \mathrm{cmx} 10 \mathrm{~cm}$, para facilitar o preparo e o estoque.

As peças de peritônio foram colocadas em frascos contendo solução de glicerina hipertônica, fechados e estocados por um prazo de 45 dias, quando então foram considerados prontos para uso (Figura 1).

Os animais foram submetidos à anestesia geral [cloridrato de quetamina ( $35 \mathrm{mg} . \mathrm{Kg}^{-1}$ ) e cloridrato de xilazina (10mg. Kg $\left.{ }^{-1}\right)$, via IM] e mantidos em ventilação espontânea durante todo o procedimento. Nos animais dos grupos 1 e 2 produziu-se um defeito retangular completo na parede abdominal anterior do animal (20mmx $15 \mathrm{~mm})$, através da exérese de um retalho músculo-aponevrótico e imediata correção com a tela cirúrgica, devidamente ajustada e fixada com pontos separados com fio de categute cromado 5-0. Após o implante do material, a pele foi suturada com fio monofilamentar de poliamida 5.0, em sutura contínua, com os nós voltados para o seu interior.

Os 17 animais do Grupo 3 (controle) foram apenas anestesiados, submetidos à depilação e não foram submetidos ao ato operatório. Foram observados concomitantemente com os animais dos dois outros grupos.

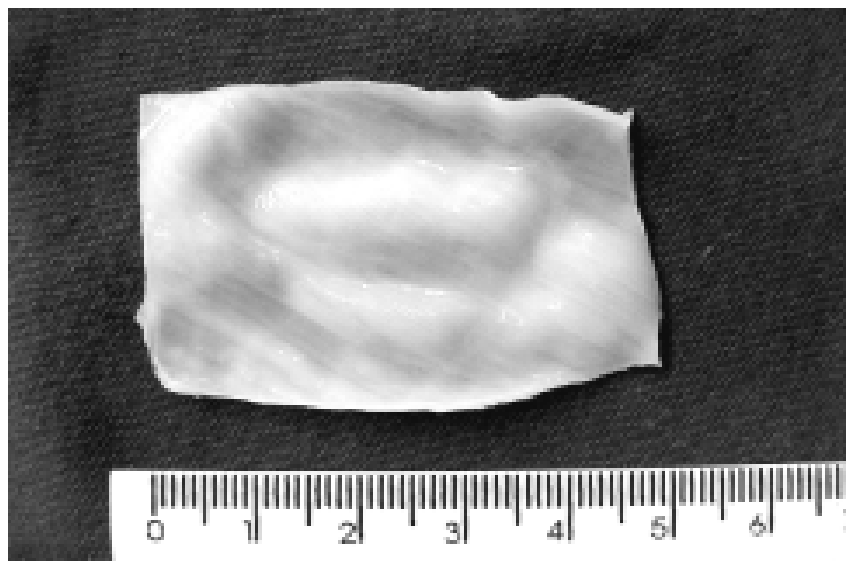

Figura 1 - Foto representativa de um pedaço de peritônio bovino pronto para uso.
Após observação dos períodos definidos, a morte foi induzida com injeção de dose letal dos anestésicos, de modo a não causar dor ou desconforto aos animais.

Para se obter o material de estudo, uma incisão em "U" foi realizada na parede abdominal anterior a fim de se retirar a pele e expor a área do implante. Retirou-se, então, uma peça contendo musculatura e a tela cirúrgica implantada (nos Grupos 1 e 2). Nos animais do Grupo 3, extraiu-se um segmento da parede abdominal semelhante ao descrito para os Grupos 1 e 2, com a finalidade de constituição de corpo de prova para estudo de resistência tênsil. Uma tira central de aproximadamente $1 \mathrm{~cm}$ de largura por $10 \mathrm{~cm}$ de comprimento deu origem ao corpo de prova para o teste de resistência tênsil. Estas peças foram envoltas em papel alumínio e folha plástica transparente, identificadas e congeladas em "freezer" comum no Laboratório de Fisiologia da FAMEMA, a uma temperatura em torno de 10 graus Celsius negativos, para posterior realização dos testes de resistência à tração.

Para se mensurar a resistência tênsil, aplicou-se o corpo de prova em máquina de ensaio universal de acionamento eletromecânico ( EMIC equipamentos e sistema de ensaio Ltda.) no Laboratório de Cirurgia Experimental da Faculdade de Medicina da UNESP-Botucatu. O corpo de prova, constituído de musculatura abdominal normal nos dois lados (direito e esquerdo) e pela tela cirúrgica implantada ao centro, foi preso perpendicularmente, após descongelamento rápido, às garras de alumínio da máquina de ensaios de tal forma que as mesmas ficassem atadas somente à musculatura normal da parede abdominal, permitindo que a tela cirúrgica implantada ficasse livre e que a linha de sutura ficasse disposta paralelamente às garras (Figura 2). A distância deixada entre as garras foi de $5 \mathrm{~cm}$. A célula de carga utilizada foi a de $50 \mathrm{~N}$, em uma velocidade de ensaio calibrada no aparelho de $30 \mathrm{~mm} / \mathrm{min}$ e o registro gráfico do estiramento foi processado em um computador equipado com um programa específico (Mtest $®)$ ). O teste foi considerado positivo quando a ruptura ocorreu na porção da interface entre a tela ou na musculatura normal. $\mathrm{O}$ valor da força aplicada, em Newton, necessária para romper o corpo de prova foi denominado força de ruptura. Nos animais do grupo 3, foi considerada força de ruptura a força necessária para romper o corpo de prova em qualquer segmento.

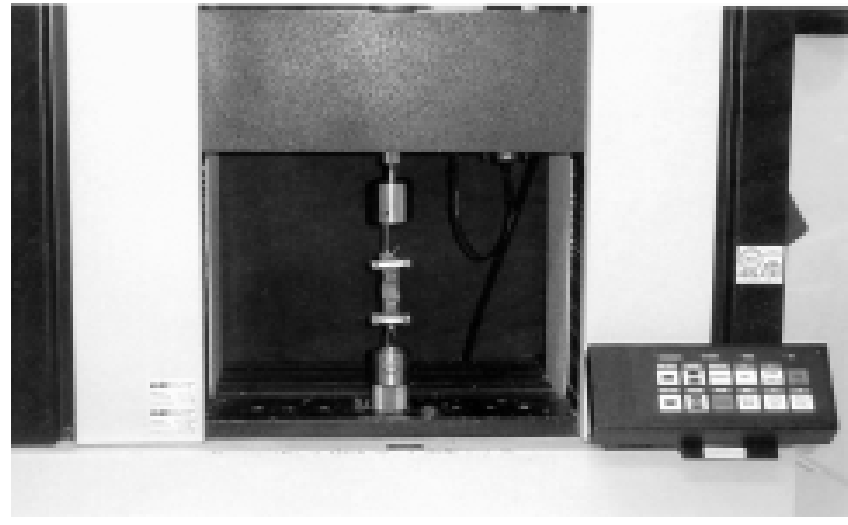

Figura 2 - Foto mostrando o corpo de prova posicionado na Máquina Universal de Ensaios antes da realização do teste de resistência à tração. 
Tabela 1 - Ratos dos grupos 1, 2 e 3 segundo os valores da resistência à tração (Newton), observados nos períodos do $7^{\circ}$ e $28^{\circ}$ dias de pós-operatório.

\begin{tabular}{lcccccc}
\hline & \multicolumn{2}{c}{ Grupo 1 (peritônio) } & \multicolumn{2}{c}{ Grupo 2 (tela) } & \multicolumn{2}{c}{ Grupo 3 (músculo) } \\
\hline & $\mathbf{7}^{\mathbf{0}} \mathbf{P O}$ & $\mathbf{2 8}^{\mathbf{0}} \mathbf{P O}$ & $\mathbf{7}^{\mathbf{0}}$ PO & $\mathbf{2 8}^{\mathbf{0}}$ PO & $\mathbf{7}^{\mathbf{0}}$ PO & $\mathbf{2 8}^{\mathbf{0}}$ PO \\
& 5,97 & 10,8 & 5,54 & 10,30 & 13,05 & 19,4 \\
& 6,51 & 12,2 & 5,75 & 14,01 & 13,88 & 19,7 \\
& 7,75 & 12,6 & 5,87 & 14,41 & 17,45 & 20,5 \\
& 8,59 & 13,1 & 8,99 & 15,64 & 19,25 & 20,8 \\
& 9,93 & 14,6 & 10,86 & 16,19 & 19,85 & 22,7 \\
& 12,01 & 18,4 & 12,64 & 16,84 & 20,65 & 26,1 \\
& 13,87 & 21,6 & 12,71 & 17,02 & 20,76 & 27,7 \\
& 17,00 & 22,1 & 12,97 & 17,06 & 21,28 & 31,1 \\
& 19,53 & 23,8 & 14,31 & 18,81 & 23,98 & \\
Média & 21,80 & & 18,46 & 19,16 & & \\
Mediana & 12,30 & 16,57 & 10,81 & 15,94 & 18,91 & 23,49 \\
\hline
\end{tabular}

Grupo peritônio vs. polipropileno vs. normal no $7^{\circ} \mathrm{PO}: p=0.003 *($ Grupo 1 e $2<3)$

Grupo peritônio vs.polipropileno vs. normal no $28^{\circ} \mathrm{PO}: \mathrm{p}=0.003 *($ Grupo 1 e $2<3$ )

Grupo 1 no $7^{\circ} \mathrm{PO}$ vs. $28^{\circ} \mathrm{PO}: \mathrm{p}=0.080(\mathrm{NS})$

Grupo 2 no $7^{\circ} \mathrm{PO}$ vs. $28^{\circ} \mathrm{PO}: \mathrm{p}=0.005^{*}\left(7^{\circ} \mathrm{PO}<28^{\circ} \mathrm{PO}\right)$

Grupo 3 no $7^{\circ} \mathrm{PO}$ vs. $28^{\circ} \mathrm{PO}: \mathrm{p}=0.074(\mathrm{NS})$

O estudo estatístico foi realizado da seguinte forma:

1) Teste de Mann-Whitney com a finalidade de comparar os ratos do $7^{\circ}$ e $28^{\circ}$ dias nos grupos 1 (peritônio), 2 (tela) e 3 (músculo) separadamente em relação aos valores de resistência à tração.

2) Análise de variância de Kruskal-Wallis para comparar as variáveis estudadas entre os grupos 1,2 e 3 para o $7^{\circ}$ e $28^{\circ}$ dias de pós-operatórios separadamente.

3) Valores com significância estatística foram considerados quando $\mathrm{p}<0,05$.

\section{RESULTADOS}

Os resultados obtidos com o teste de tração e expressos na Tabela 1 demonstram que tanto no $7^{\circ}$ como no $28^{\circ}$ PO dos grupos 1 e 2, os valores médios não diferiram, mostrando um comportamento semelhante na força tênsil entre o peritônio parietal bovino e a tela de polipropileno. Ambos os grupos, entretanto, apresentaram valores menores de resistência à tração quando comparados com a parede abdominal normal (Grupo 3).

Quando se comparou o $7^{\circ}$ e o $28^{\circ} \mathrm{PO}$ dentro de um mesmo grupo os valores médios da resistência à tração do subgrupo B $\left(28^{\circ} \mathrm{PO}\right)$ foram maiores que no subgrupo A ( $7^{\circ}$ $\mathrm{PO}$ ), sendo estatisticamente significante no Grupo 2. Com o tamanho da amostra utilizada, a aplicação de teste de MannWhitney não demonstrou significância estatística nos Grupos 1 e 3 , embora com uma tendência para tal, visto que o " $z$ " calculado é muito próximo do "z" crítico.

\section{DISCUSSÃO}

Um menor índice de recidivas têm propagado o uso de próteses na correção de hérnias abdominais ${ }^{3,4,22}$. Experi- mentos em animais de laboratório têm envolvido a mensuração da resistência do material implantado ${ }^{23,24}$, devido à necessidade de se testar a capacidade de suportar as tensões às quais será submetido, caso venha a ter uso clínico.

A unidade de expressão da resistência tênsil é variável na literatura biomédica. A forma de expressão mais fidedigna é o valor da força de carga aplicada sobre um valor de área. Contudo, para que esta unidade expresse a real força tênsil, há necessidade de se estabelecer duas condições: o formato dos corpos de prova deve direcionar o centro da força aplicada e sua área neste centro deve ser conhecida e/ou padronizada. Neste experimento a largura e o comprimento dos corpos de prova foram semelhantes entre si, contudo as espessuras foram variáveis. Além disso, eles foram retangulares, não sendo possível, dado às características do experimento, constituir corpos de provas na forma de ampulheta. Por este motivo, demonstrou-se a resistência à tração como o valor máximo registrado pela Máquina Universal de Ensaios no momento da ruptura dos mesmos, tida como uma forma de expressão adequada ${ }^{23,5}$. Não há um padrão na forma de expressar esta capacidade de resistência à tensão, o que se constitui em uma variável interveniente que dificulta a comparação entre os trabalhos ${ }^{25-28}$.

Em que pese estes obstáculos, o fato é que a submissão de corpos de prova de materiais a uma tração é um teste conceituado e considerado como válido na Engenharia para medir resistência de materiais usados nas mais diversas áreas da indústria e, portanto, por similaridade pôde ser considerado adequado aos propósitos desta pesquisa.

Há relatos antigos do uso do peritônio parietal bovino conservado como xenoimplante em cães e em seres humanos, porém sob outras condições de preparo, conservação e aplicação ${ }^{29,30}$. Tais condições não encontram mais suporte diante do conhecimento científico atual. Em medicina veteri- 
nária foi utilizado na substituição de um retalho diafragmático de cães. Embora tenha sido relatada boa integração do mesmo, o estudo não incluiu teste de resistência à tração, apenas análise de aspectos histológicos ${ }^{20}$. Estes mesmos autores relataram bons resultados em uma série de casos clínicos (correção de hérnias perineais em cães) ${ }^{21}$.

Os peritônios de suínos, caninos e equiinos já foram comparados com o de bovinos e concluiu-se que este último é o que suporta melhor a resistência à tração e a conservação tecidual em glicerina hipertônica à temperatura ambiente não prejudicou esta mesma resistência ${ }^{31}$. Nesta pesquisa, todos os corpos de prova dos Grupos 1 e 2 romperam-se na linha de sutura, zona reconhecidamente mais frágil que a parede abdominal normal e que a própria tela cirúrgica. Há relatos de resultados semelhantes ${ }^{32,5}$, entretanto isto não se constitui em uma uniformidade, pois há estudos de resistência tênsil com materiais biológicos cuja ruptura se deu predominantemente na parede abdominal normal ou na própria área de implante, e não na interface cicatricial ${ }^{32,24}$.

É relevante na literatura biomédica, relato clínico de recidivas herniárias ditas centrais, ou seja, na área da prótese implantada ${ }^{32,33}$. Pode-se aceitar esta situação como um dado de ineficácia da prótese. $\mathrm{O}$ fato da tela de peritônio bovino não ter se rompido em nenhum teste permite inferir que a sua resistência é compatível com o esperado dos materiais protéticos utilizados nas correções de hérnias abdominais. Os dois grupos com o uso de próteses mostraram um resultado final de resistência à tração menor que a parede abdominal normal do rato. $\mathrm{O}$ achado está de acordo com o paradigma de que um tecido cicatricial, por maior força tênsil que adquira, sempre será inferior ao tecido normal que ele está reparando.
Nos diferentes períodos de observação dentro dos próprios grupos, os valores médios obtidos no subgrupo $\mathrm{B}$ $\left(28^{\circ} \mathrm{PO}\right)$ foram maiores que os do subgrupo A ( $\left.7^{\circ} \mathrm{PO}\right)$, sendo que somente no Grupo 2 (polipropileno) estes valores foram estatisticamente significantes. Nos demais grupos, 1 (peritônio) e 3 (músculo), o tamanho das amostras não permitiu rejeitar a hipótese de nulidade, embora os números apontassem para tal.

É possível inferir que, mesmo no Grupo 1, os valores médios da resistência à tração nos subgrupos $\mathrm{B}$ foram maiores que os dos subgrupos $\mathrm{A}$, revelando um ganho de força tênsil com a evolução do processo cicatricial e de integração dos materiais estudados.

Com isso, faz-se oportuno a elucidação de outros tópicos relacionados ao uso do peritônio bovino como tela cirúrgica na correção de hérnias ventrais. Seu processo de absorção, sua relação com a formação de aderências, sua utilização na presença de infecção e a investigação de aspectos específicos de rejeição são alguns exemplos. Tais experimentos podem valer-se de um período de observação mais prolongado, do uso de outros animais de experimentação e da aplicação em outros modelos para correção de hérnias.

\section{AGRADECIMENTOS}

1. À Coordenação de Aperfeiçoamento de Pessoal de Nível Superior (Capes) pelo incentivo e financiamento desta pesquisa

2. Ao Laboratório de Cirurgia Experimental da Faculdade de Medicina da UNESP-Botucatu, pela oportunidade de realizar os testes de resistência à tração.

\begin{abstract}
Objective: The aim of this study is to assess the tensile strength of the preserved bovine peritoneum (PBP) as a surgical mesh in rats ventral hernia. Methods: Using 57 rats, two mesh materials, the PBP and polypropylene (PP), were compared after implantation in a standardized ventral hernia model (full-thickness abdominal wall defects). After 7 (subgroup A) and 28 ( subgroup B) days of implantation, they were removed and tensile strength assessment "in vitro" was performed. One group, with no procedure, just normal abdominal wall, was used as control for tensile strength. The Mann-Whitney and KruskalWallis tests were applied for statistical analysis. Results: All animals survived until the end point, but one (PBP group, subgroup $B)$. No recurrence was seen during the period of observation. The mean load required to disrupt the interface were similar in PBP and PP groups, and both were weaker than normal group $(p=0,003)$ in all periods of observation. Conclusion: Preserved bovine peritoneum is a biological mesh that can be used for ventral hernia repair in rat model.
\end{abstract}

Key words: Hernia, Ventral; Surgical mesh; Peritoneum; Models, Animal.

\section{REFERÊNCIAS}

1. Usher FC, Wallace AS. Tissue reaction to plastics. Arch Surg. 1958; 76(6):997-9

2. Lichtenstein IL, Shulman AG, Amid PK, et al. The tension-free hernioplasty. Am J Surg.1989;157(2):188-93.

3. Scott NW, McCormack K, Graham P, et al. Open mesh versus non-mesh repair of femoral and inguinal hernia. Cochrane Database Syst Rev. 2002, (4) pCD002197

4. Pavlidis TE, Atmatzidis KS, Lazaridis CN, et al. Comparison between modern mesh and conventional non-mesh methods of inguinal hernia repair. Minerva Chir. 2002; 57(1):7-12.
5. Kama NA, Coskun T, Yavuz H, et al. Autologous skin graft, human dura mater and polypropylene mesh for the repair of ventral abdominal hernias: an experimental study. Eur J Surg. 1999; 165(11):1080-5.

6. Baykal A, Yorganci K, Sokmensuer C, et al. An experimental study of the adhesive potential of different meshes. Eur J Surg. 2000; 166(6):490-4.

7. Zachariou Z. Amniotic membranes as prosthetic material: experimental utilization data of a rat model. J Pediatr Surg. 1997;32(10):1458-63.

8. Szabo A, Haj M, Waxsman I, et al. Evaluation of seprafilm and amniotic membrane as adhesion prophylaxis in mesh repair of abdominal wall hernia in rats. Eur Surg Res. 2000;32(2):125-8. 
9. Cataldo MLS, Silva Al, Guerra AJ. Emprego do saco herniário na correção cirúrgica das hérnias incisionais longitudinais - aspectos experimentais no cão. Rev Col Bras Cir. 1981;8(4):167-70.

10. Silva AL. Uso de saco herniário no reforço da hernioplastia inguinal [nota prévia]. Rev Col Bras Cir, 1995;22(3):153-4.

11. Gruen RL, Morrison WA, Vellar ID. The tensor fasciae latae myocutaneous flap closure of major chest and abdominal wall defects. Aust N Z J Surg. 1998;68(9): 666-9.

12. Koshima I, Moriguchi $\mathrm{T}$, Inagawa $\mathrm{K}$, et al. Dynamic reconstruction of the abdominal wall using reinnervated free rectus femoris muscle transfer. Ann Plast Surg. 1999; 43(2):199-203.

13. Prevel CD, Eppley BL, Summerlin DJ, et al. Small intestinal submucosa: utilization for repair rodent abdominal wall defects. Ann Plast Surg. 1995; 35(4):374-80.

14. Clarke KM, Lantz GC, Salisbury SK, et al. Intestine submucosa and polypropylene mesh for abdominal wall repair in dogs. J Surg Res. 1996; 60(1):107-14.

15. Abraham GA, Murray J, Billiar, K et al. Evaluation of the porcine intestinal collagen layer as a biomaterial. J Biomed Mater Res. 2000; 51(3):442-52.

16. Steinau G, Dreuw B, Schleef J et al. [Diaphragm replacement - an experimental animal study]. Langenbecks Arch Chir. 1997; 382(2):74-8

17. Kapan S, Kapan M, Goksoy E, et al. Comparison of PTFE, pericardium bovine and fascia lata for repair of incisional hernia in rat model, experimental study. Hernia. 2003; 7(1):39-43

18. Reyes EEF. Implante de pericárdio de eqüino preservado em glicerina a $98 \%$ como substituto biológico de segmento de tendão de músculo extensor ulnar do carpo em cães - estudo experimental [dissertação]. São Paulo (SP): Universidade de São Paulo; 1998.

19. Parizek J, Husek Z, Mericka P, et al. Ovine pericardium: a new material for duraplasty. J Neurosurg. 1996; 84(3):508-13

20. Daleck CR, Alessi AC, Costa-Neto JM, et al. Substituição de um retalho diafragmático de cão por peritônio de bovino conservado em glicerina: estudo experimental. Ars Vet. 1988; 4(1):53-61.

21. Daleck CR, Daleck CLM, Padilha-Filho JG, et al. Reparação de hérnia perineal em cães com peritônio de bovino conservado em glicerina. Cienc Rural. 1992; 22(2):179-83.

22. Cassar K, Munro A. Surgical treatment of incisional hernia. Br J Surg. 2002; 89(5):534-45.

23. Morrell AC. Estudo da integração e da força de ruptura da prótese de colágeno bovino implantada em defeito provocado na parede abdominal de coelhos [dissertação]. São Paulo (SP): Universidade Federal de São Paulo; 1993.
24. Quintana LFC, Czeczko NG, Cuenca RM, et al. Avaliação da biocompatibilidade das telas de polipropileno, poliglactina $910 \mathrm{e}$ poliéster no reparo de defeitos da parede abdominal ventral. ABCD Arq Bras Cir Dig. 2002;15(4):148-52.

25. Lamb JP, Vitale T, Kaminski DL. Comparative evaluation of synthetic meshes used for abdominal wall replacement. Surgery. 1983;83(5):643-8.

26. Tyrell J, Silberman H, Chandrasoma P, et al. Absorbable versus permanent mesh in abdominal operations. Surg Gynecol Obstet. 1989;168(3):227-32.

27. Hooker GD, Taylor BM, Driman DK. Prevention of adhesion formation with use of sodium hyaluronate-based bioresorbable membrane in a rat model of ventral hernia repair with polypropylene mesh - a randomized, controlled study. Surgery. 1999;125(2):211-6.

28. Le Blanc KA, Bellanger D, Rhynes KV, et al. Tissue attachment strength of prosthetic meshes used in ventral and incisional hernia repair. A study in the New Zealand white rabbit adhesion model. Surg Endosc. 2002;16(11):1542-6.

29. Eliseev NT, Pashkova LA. [Pathomorphological changes in heterogenic peritoneum in plastic reconstruction of the anterior abdominal wall (experimental study)]. Klin Khir. 1968; 8:30-4.

30. Yeliseyev NT. Application of preserved heteroperitoneum for plastic operation of postoperational ventral hernia. Acta Chir Plast.1970; 12(2):77-84.

31. Batista LC, Daleck CR, Shimano AC, et al. Estudo comparativo da resistência à tração do peritônio (bovino, eqüino, suíno e canino) a fresco e conservado em glicerina. Braz J Vet Res Anim Sci. 1996; 33(suppl.):305-12.

32. Bellón JM, Buján J, Contreras LA, et al. Comparison of a new type of polytetrafluoroethylene patch (Mycro Mesh) and polypropylene prosthesis (Marlex) for repair of abdominal wall defects. J Am Coll Surg. 1996; 183(1):11-8.

Endereço para correspondência:

Eduardo Lemos de Souza Bastos

Rua Carlos Botelho, 246.

Maria Izabel

17515-240- Marília - SP

edbastos@unimedmarilia.com.br 